Europhys. Lett., 5 (4), pp. 355-360 (1988)

\title{
Critical Behavior of the Bethe Lattice Spin Glass.
}

\author{
J. M. Carlson $\left(^{*}\right)$, J. T. Chayes $(* *)$, L. Chayes $\left(^{* *}\right)$, J. P. Sethna $\left(^{* * *}\right)$ \\ and D. J. Thouless $\left(* *^{*}\right)$ \\ (*) Laboratory of Atomic and Solid State Physics \\ Cornell University, Ithaca, N.Y. 14853 \\ (**) Department of Mathematics, University of California, Los Angeles, CA 90024 \\ (***) Laboratory of Atomic and Solid State Physics \\ Cornell University, Ithaca, N.Y. 14853 \\ (**) Physics Department, FM-15, University of Washington, Seattle, WA 98195
}

(received 26 October 1987; accepted 27 November 1987)

PACS. 75.50K - Amorphous magnetic materials.

PACS. 64.60 - General studies of phase transitions.

PACS. 02.30 - Function theory, analysis.

\begin{abstract}
We present results of a rigorous analysis of the $\pm J$ Ising spin glass on the Bethe lattice with uncorrelated boundary conditions. We derive phase diagrams as functions of temperature vs. percentage of ferromagnetic bonds, and, when half of the bonds are ferromagnetic, temperature vs. external field. Critical exponents are also determined. Using bifurcation theory, we establish the existence of nontrivial distributions of single-site magnetizations within the low-temperature/small-field phase boundaries; these solutions reflect spin glass, ferromagnetic and magnetized spin glass behavior.
\end{abstract}

We have studied the $\pm J$ Ising spin glass model on the Bethe lattice with varying fractions of ferromagnetic bonds [1-3]. Here we present concise arguments for results which have been proven rigorously elsewhere $[2,3]$. Recursion relations for the distribution of single-site magnetizations are derived and studied as a dynamical system on an appropriate function space. Bulk properties are described by attractors of the recursion relations, and the phase transitions correspond to bifurcations in the dynamics. Using these methods, we derive the phase diagram as a function of temperature and fraction of ferromagnetic bonds, determine critical exponents at the phase boundaries, and prove the existence of a stable spin glass solution near the high-temperature paramagnetic phase boundary. At least in terms of single-site properties, the existence proof precludes chaos, an infinite hierarchy of transitions, and other bizarre possibilities. We also prove that (when half of the bonds are ferromagnetic) the spin glass transition persists in the presence of small external magnetic fields, and derive the asymptotics of the de Almeida-Thouless (AT) line [4].

The Bethe lattice provides a useful alternative to the more commonly studied infiniterange Sherrington-Kirkpatrick (SK) model of spin glasses [5]; here the interactions are short-range, the analysis is straightforward and the results have been made completely 
rigorous. In spite of differences between the models, our phase diagrams agree with those of ref. [4] on the "replica symmetric» solution of the SK model. Indeed, our solution is also reminiscent of the replica-symmetric solution, although here we can show that it is the correct solution for the Bethe lattice with statistically uncorrelated boundary conditions. The possibility of other boundary conditions will be briefly discussed at the end of this letter.

The Hamiltonian typically used to study spin glasses is the Edwards-Anderson Hamiltonian [6]

$$
\mathscr{K}=-\sum_{\langle i, j\rangle} J_{i, j} \sigma_{i} \sigma_{j}-\sum_{i} H_{i} \sigma_{i}
$$

The nearest-neighbor bonds $J_{i, j}$ are of equal strength but of random sign: $J_{i, j}=J \theta_{i, j}$, where the $\theta_{i, j}$ s are quenched and independently distributed according to

$$
\theta_{i, j}=\left\{\begin{array}{l}
+1, \text { with probability } \lambda \\
-1, \text { with probability } 1-\lambda,
\end{array}\right.
$$

and $H_{i}$ is the external field at site $i$, which may or may not be uniform.

The analysis in this paper is performed on the half-space $\left(^{1}\right)$ Bethe lattice with forward branching ratio 2 . The treelike structure allows us to write explicit recursion relations giving properties of level $n$ in terms of those of level $n+1$. For example, the magnetization of the origin, $\left\langle\sigma_{x}\right\rangle$, is given by

$$
\left\langle\sigma_{x}\right\rangle=\frac{p\left(\theta_{x, y}\left\langle\sigma_{y}\right\rangle+\theta_{x, z}\left\langle\sigma_{z}\right\rangle\right)+h_{x}\left(1+p^{2} \theta_{x, y}\left\langle\sigma_{y}\right\rangle \theta_{x, z}\left\langle\sigma_{z}\right\rangle\right)}{1+p^{2} \theta_{x, y}\left\langle\sigma_{y}\right\rangle \theta_{x, z}\left\langle\sigma_{z}\right\rangle+h_{x} p\left(\theta_{x, y}\left\langle\sigma_{y}\right\rangle+\theta_{x, z}\left\langle\sigma_{z}\right\rangle\right)},
$$

where $p=\operatorname{tgh}(J / k T), h_{i}=\operatorname{tgh}\left(H_{i} / k T\right), y$ and $z$ are the sites connected to the origin, and the angle brackets denote thermal averages $\left({ }^{2}\right)$.

Boundary conditions correspond to initial conditions for iteration of the recursion relation (3). Therefore, since most of the sites of a tree lie near the boundary, naive averages would be skewed by surface transients. We extract bulk behavior by performing our calculations on a large finite tree of radius $R_{1}$ embedded in an even larger lattice of radius $R_{2}$, and taking $R_{2} \rightarrow \infty$ before $R_{1} \rightarrow \infty$. Bulk properties are thus determined by the behavior of the recursion relation after many iterations-the attractor. We study the stable self-consistent fixed point, which we believe should describe most uncorrelated initial conditions (all but a set of measure zero) $\left(^{3}\right)$. Frustration initially associated with the boundary is transmitted inwards, which leads to the glassy behavior.

(') On the Bethe lattice it is easier to calculate half-space quantities, treating a particular site (say $x$ ) as the origin, and considering only the forward branching part of the lattice. Once the calculation is complete, a simple formula relates the full-space quantities to those that were calculated for the partial lattice. See ref. [2].

$\left(^{2}\right)$ Here $\left\langle\sigma_{y}\right\rangle$ and $\left\langle\sigma_{z}\right\rangle$ are the magnetizations sites $y$ and $z$ would have if they were disconnected from the site $x$.

$\left({ }^{3}\right)$ What we have shown rigorously is that our solution has a positive basin of attraction in the space of square-integrable functions. There are, however, a huge number of possible self-consistent solutions, corresponding to unlikely boundary conditions. For example, if one makes a gauge transformation to make all of the bonds ferromagnetic, and chooses «up" boundary conditions in that gauge, one has a Mattis spin glass - essentially a ferromagnet. (In an external field, this is the random field Ising model on the Bethe lattice and has been studied by Bruinsma [7].) 
At $h_{i} \equiv 0$, the paramagnetic phase boundary is determined by a moment analysis of eq. (3). For convenience, we first define $X=\theta\left\langle\sigma_{x}\right\rangle$, etc., where $\theta$ is the sign of the bond joining the origin to a third neighbor in the «full tree». Then the random variables $X, Y$ and $Z$ satisfy

$$
X=\frac{p \theta(Y+Z)}{1+p^{2} Y Z},
$$

with $Y$ and $Z$ independent and identically distributed, and $«=»$ to be understood in the sense of distributions. The corresponding equation for the probability density, $\rho(X)$, of $X$ is $\rho(X)=\int \rho(Y) \rho(Z) \delta(X-F(Y, Z ; \theta))$, where $F(Y, Z ; \theta)$ is the r.h.s. of eq. (4). Up to multiplicative constants, the first moment, $m \equiv \bar{X}=\int X_{p}(X)$, is the magnetization, which is the order parameter for the ferromagnet; the second moment, $q_{\mathrm{EA}} \equiv \bar{X}^{2}=\int X^{2} \rho(X)$, is the Edwards-Anderson order parameter for the spin glass. Clearly the paramagnetic solution $X=Y=Z \equiv 0$ is a fixed point of the recursion relation for all values of $p$ and $\lambda$. As the temperature is lowered, this solution becomes unstable and a phase transition occurs. Linear analysis-performed by discarding the denominator in eq. (4)-indicates that an infinitesimal first moment will contract if $2 p(2 \lambda-1)<1$. Similarly, $q_{\mathrm{EA}}=0$ is stable provided $2 p^{2}<1$. The intersection of these two regions is the paramagnetic phase, where a detailed analysis shows that a general class of initial distributions iterates to $X \equiv 0$ (i.e. $p(X)=\delta(X))$. See fig. 1 .

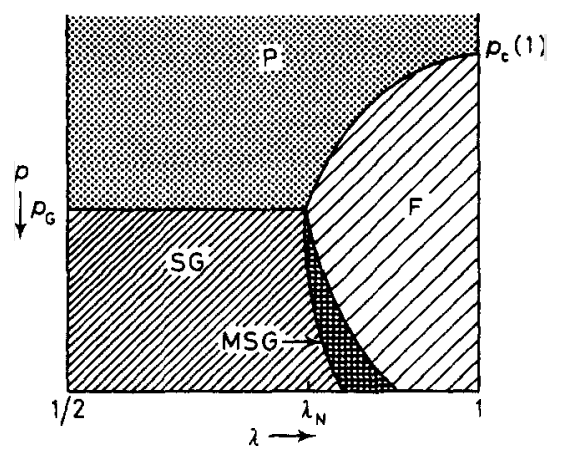

Fig. 1. - Phase diagram as a function of temperature $(p=\operatorname{tgh}(J / k T))$ and fraction of ferromagnetic bonds, $\lambda$. At high temperatures, the system is paramagnetic (P). As temperature decreases, not too surprisingly we find a ferromagnetic $(F)$ phase near $\lambda=1$. More surprisingly, both the transition temperature, $p=1 / \sqrt{2} \equiv p_{\mathrm{G}}$, and the character of the spin glass (SG) phase remain constant from $\lambda=1 / 2$ until $\lambda=1 / 2(1+1 / \sqrt{2}) \equiv \lambda_{N} \approx 85 \%$. Intermediate between the $(F)$ and (SG) phases is a magnetized spin glass (MSG) phase which shares properties of both. Phase diagram for $\lambda<1 / 2$ is given by reflection across the line $\lambda=1 / 2$, with analogues of the magnetized phases exhibiting antiferromagnetic order. Zero temperature intercepts are from ref. [8].

Moment analysis in the low-temperature phase(s)-studying the growth of $m$ or $q_{\mathrm{EA}}$ when $2 p(2 \lambda-1)>1$ or $2 p^{2}>1$-can be used to produce upper and lower bounds on the order parameters. See, e.g., fig. 2. This gives the critical exponents $\beta=1 / 2$ for $m$ at the ferromagnetic transition and $\beta=1$ for $q_{\mathrm{EA}}$ at the spin glass transition. The scaling behavior is more complex near the multicritical point $\left(p_{G}, \lambda_{N}\right)$ where the spin glass and ferromagnetic boundaries intersect. It is worth noting that the Nishimori line [9] $p=2 \lambda-1$ passes through the multicritical point. 


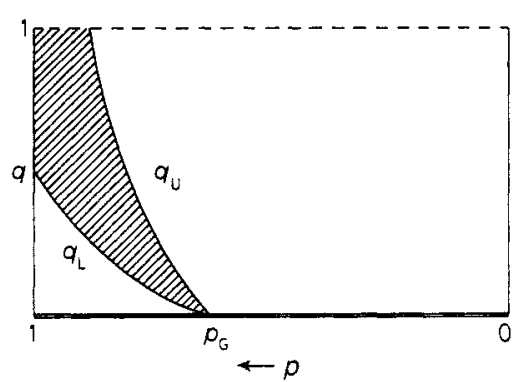

Fig. 2. - Bounds on the spin glass order parameter. A finite distance in from the boundary of the Bethe lattice, any nonzero initial distribution of magnetizations will give rise to a distribution whose second moment lies within the shaded band $\left(0<q_{\mathrm{L}}<q_{\mathrm{EA}}<q_{\mathrm{U}}\right)$ for any $\lambda<\lambda_{\mathrm{N}}$. The slopes of the upper and lower bounds agree at the critical point; there is a second-order transition with $q_{\mathrm{EA}} \sim 2\left|p-p_{\mathrm{G}}\right| / p_{\mathrm{G}}$.

Between the ferromagnet and the spin glass, we find a magnetized spin glass (MSG) phase. The transition to this phase from the spin glass is marked by the onset of nonzero magnetization; near the multicritical point, we determine the phase boundary: $\sqrt{p_{\mathrm{G}}}\left|p-p_{\mathrm{G}}\right| \sim \sqrt{\left|\lambda-\lambda_{\mathrm{N}}\right|}$ by moment analysis. On the other hand, the boundary between the MSG and the ferromagnet cannot be derived via moment analysis; all moments are smooth through this transition. Nevertheless, the approach to the transition from the ferromagnetic phase is signaled by divergence of a susceptibility conjugate to $q_{\mathrm{EA}}$ :

$$
\chi \mathrm{EA}=\sum_{j} \overline{\left\langle\sigma_{x} \sigma_{j}\right\rangle^{2}-\left\langle\sigma_{x}\right\rangle^{2}\left\langle\sigma_{j}\right\rangle^{2}}
$$

In order to calculate $\chi_{\mathrm{EA}}$ on the Bethe lattice, we studied a rather complicated set of coupled recursion relations for two copies of the same quench of the lattice which are ferromagnetically joined by bonds of strength $r$. Provided that the spins on the upper and lower replicas obey the constraint $\left\langle\sigma_{x}^{U}\right\rangle=\left\langle\sigma_{x}^{\mathrm{L}}\right\rangle$ (as is the case in the ferromagnetic phase), $\chi_{\mathrm{EA}}=\left.(\mathrm{d} / \mathrm{d} r) \overline{\left\langle\sigma_{x}^{\mathrm{U}} \sigma_{x}^{\mathrm{L}}\right\rangle}\right|_{\mathrm{r}=0}$. Using this, we have shown that $\chi_{\mathrm{EA}}$ diverges with critical exponent $\gamma=1$ along the asymptotic curve $\sqrt{3 p_{\mathrm{G}}}\left|p-p_{\mathrm{G}}\right| \sim \sqrt{\left|\lambda-\lambda_{\mathrm{N}}\right|}$, marking the MSG-ferromagnetic boundary.

For uniform $h=\left|h_{i}\right| \neq 0$ and $\lambda=1 / 2$, we derive the AT line by methods identical to our analysis of the MSG-ferromagnetic transition, with similar results. Namely, we find that all moments of $p(X)$ are smooth as a function of $h$. Nevertheless, divergence of $\chi_{E A}$ signals a transition between a paramagnet at large fields and a spin glass phase at small fields, with asymptotic phase boundary $\left(32 p_{\mathrm{G}}\right)^{1 / 3}\left|p-p_{\mathrm{G}}\right| \sim h^{2 / 3}$. Again we have $\gamma=1$. There are, however, anomalies in the crossover behavior at the transition $\left({ }^{4}\right)$.

Next let us address the questions of existence and nature of solutions in the lowtemperature phases. We begin by deriving the scaling form of $f$ at the paramagnetic phase boundary. Moment analysis (see fig. 2) indicates that the random variables should be scaled according to $X^{*}=X / \sqrt{\Delta}$, where $\Delta$ measures the distance from the critical line (e.g. $\Delta=p-p_{\mathrm{G}}$ ). Along the phase boundary, the rescaled recursion relation (4) takes the linear form

$$
X^{*}=p \theta\left(Y^{*}+Z^{*}\right)
$$

$\left({ }^{4}\right)$ Calculations based on naive definitions of a crossover exponent, $\varphi$, yield a value of $\varphi=2$ for approach to the transition along the line $p=p_{\mathrm{G}}, h \rightarrow 0$, whereas $p=3$ for approach to the AT line. 


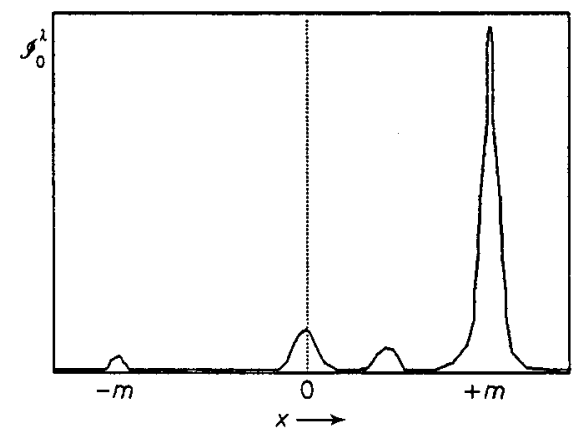

Fig. 3. - Scaling form of $\mathscr{J}_{0}^{\hat{\lambda}}$ near the pure ferromagnet $(\lambda \approx 0.99)$, numerically generated by iteration of eq. (6).

For the spin glass transition (where $\lambda<\lambda_{\mathrm{N}}$ and $p=p_{\mathrm{G}} \equiv 1 / \sqrt{2}$ ), (6) is satisfied by a Gaussian $\left(^{5}\right) \rho\left(X^{*}\right)=\mathscr{G}_{0}\left(X^{*}\right)$ of arbitrary width, independent of $\lambda$. However, along the ferromagnetic boundary (where $\lambda>\lambda_{N}$ and $p=p_{\mathrm{c}}(\lambda) \equiv[2(2 \lambda-1)]^{-1}$ ), (6) is not satisfied by any well-known distribution. Nevertheless, we can obtain all moments by raising (6) the $n$-th power, averaging and solving inductively. Using the Hamburger moment theorems [10], we have shown that for each value of $\lambda>\lambda_{N}$, we obtain a different distribution $\rho\left(X^{*}\right)=\mathscr{J}_{0}^{\lambda}\left(X^{*}\right)$ which is unique up to the scale of the mean. See fig. 3 .

Away from points of bifurcation, small changes in parameters like temperature result in continuous changes in $f(X)$. This is guaranteed by the implicit function theorem. The recursion relation (4) gives rise to a linear operator which acts on perturbations to the fixedpoint density $f(X)$; if none of the eigenvalues of this operator are zero, the implicit function theorem gives the new $\rho$ in terms of the old. However, along the paramagnetic critical line, the rescaled recursion relation (6) is linear, so that the random variables may be multiplied by an arbitrary constant; differentiating $\rho$ with respect to this scale produces an eigenfunction with zero eigenvalue $\left({ }^{6}\right)$. At the multicritical point, two zero modes emerge; one is associated with the mean and the other with the width.

Bifurcation theory allows one to identify the new solutions at places where the implicit function theorem breaks down. The center manifold theorem [11] reduces the problem of searching function space for the new solutions to searching a certain surface (with one dimension per zero mode). One then looks at the nonlinear terms of the recursion relation to determine the solution. We find a spin glass solution for $p \geqslant p_{\mathrm{G}}$ which is stable until $\lambda \approx \lambda_{\mathrm{N}}$ and unstable thereafter, a stable magnetized distribution for $\lambda\rangle_{N}$ near $p_{c}(\lambda)$, and an interesting codimension-two bifurcation diagram at the multicritical point.

We believe that the spin glass solution for uncorrelated boundary conditions may correspond to the "replica symmetric" solution of the SK model $[4,12,13]$. Indeed, in the limit that the number of nearest neighbors $K \rightarrow \infty$, the solution apparently approaches the

(5) By the moment analysis, if $\lambda<\lambda_{N}$ it turns out that all odd moments vanish and hence $\theta$ may be ignored. Thus when $p=1 / \sqrt{2}$ and $\lambda<\lambda_{N}$, (6) reduces to the addition law for Gaussian random variables $X^{*}=\left(Y^{*}+Z^{*}\right) / \sqrt{2}$.

${ }^{6}$ ) Along the spin glass critical line, the eigenfunctions of the linear operator are Hermite polynomials multiplied by the Gaussian distribution, with eigenvalues $2 p_{\mathrm{G}}^{n}-1$ for $n$ even and $2 p_{\mathrm{G}}^{n}(2 \lambda-1)-1$ for $n$ odd. Along the ferromagnetic line, the eigenfunctions are given in terms of derivatives of the fixed point density by $\mathscr{J}_{n}=\left[\mathscr{J}_{0}\right]^{(n)}$ for $n$ even and $\left[X \mathscr{J}_{0}\right]^{(n)}$ for $n$ odd, with eigenvalues $2 p^{n}-1$ for $n$ even and $2 p^{n}(2 \lambda-1)-1$ for $n$ odd. We have shown that the $\mathscr{J}_{n}$ are entire and form a complete set in an appropriate space. 
"replica symmetric" distribution $\left({ }^{7}\right)$. Hence this work may represent only the first step in a more complete analysis. In any case, we expect that the results presented here for the MSGferromagnetic and AT lines, the paramagnetic phase boundaries and the scaling forms of the distributions of single-site magnetizations along these phase boundaries will continue to hold (by analogy with the SK model). However, because our results for the MSG-spin glass phase boundary depend on higher-order terms, the asymptotics may be somewhat altered.

The mean-field theory of spin glasses has been a longstanding, difficult problem. The infinite-range SK model took many years to understand; the analysis remains subtle and somewhat inscrutable, and the relationship to finite-dimensional systems remains unclear. The mean-field theory on the Bethe lattice is much simpler. Mathematical proofs require some care and machinery $[2,3]$; in this paper we have attempted to present these results and methods in a more pedagogical form. We hope that the relative simplicity of the analysis and the short-range interactions will allow us to better understand the implications for finitedimensional short-range spin glasses.

We thank D. Dhar, D. S. Fisher, M. E. Fisher, J. Fröhlich, J. Guckenheimer, D. Huse, H. Kesten, C. Kwon, M. Mezard, P. Mottishaw, C. M. Newman and, especially, $R$. SINGH for many useful discussions. The work of JTC was supported by the Army Research Office through a grant to the Mathematical Sciences Institute of Cornell University, that of LC by the DOE under grant DE-AC02-83ER13044, that of DJT by the NSF under grant DMR-8319301, and that of JMC and JPS by the NSF under grant DMR8503544 .

(7) As $K \rightarrow \infty$, our recursion relation apparently tends to the replica-symmetric relation: $X=\operatorname{tgh}[\alpha \sqrt{q} W]$, where $\alpha=p / \sqrt{K}, q$ is the second moment of the distribution $p(X)$, and $W$ is a Gaussian of unit width. (We thank C. M. Newman for his efforts in this derivation.) In any case, we have verified that as $K \rightarrow \infty$, the second moments $q_{k}$ of the distributions $\rho_{K}$ converge to $q$ to second order in $\alpha-\alpha_{c}$.

\section{REFERENCES}

[1] Thouless D. J., Phys. Rev. Lett., 56 (1986) 1082.

[2] Chayes J. T., Chayes L., Sethna J. P. and Thouless D. J., Commun. Math. Phys., 106 (1986) 41.

[3] Carlson J. M., Chayes J. T., Chayes L., Sethna J. P. and Thouless D. J., in preparation.

[4] De Almeida J. R. L. and Thovless D. J., J. Phys. A, 11 (1978) 983.

[5] Sherington D. and KirkPatrick S., Phys. Rev. Lett., 35 (1975) 1792.

[6] Edwards S. F. and Anderson P. W., J. Phys. F, 5 (1975) 965.

[7] Bruinsma R., Phys. Rev. B, 25 (1984) 3438.

[8] Thouless D. J. and Kwon C., in preparation.

[9] Nishimori H., J. Phys. C, 14 (1980) 4071.

[10] See e.g. ReEd M. and Simon B., Methods of Mathematical Physics, Vol. 2 (Academic Press, New York, N.Y.) 1975, pp. 145, 205.

[11] See e.g. BERger M. S., Nonlinearity and Functional Analysis (Academic Press, New York, N. Y.) 1977, Chapt. 4.1.

[12] MotTishaw P., Europhys. Lett., to appear (1987).

[13] Parisi G., Phys. Rev. Lett., 43 (1979) 1754. 